\title{
Perfect Codes Over Induced Subgraphs of Unit Graphs of Ring of Integers Modulo $n$
}

\author{
${ }^{1}$ MOHAMMAD HASSAN MUDABER, ${ }^{2}$ NOR HANIZA SARMIN, ${ }^{3}$ IBRAHIM GAMBO \\ ${ }^{1,2,3}$ Department of Mathematical Sciences, Faculty of Science \\ Universiti Teknologi Malaysia \\ 81310 UTM Johor Bahru \\ MALAYSIA
}

\begin{abstract}
The induced subgraph of a unit graph with vertex set as the idempotent elements of a ring $R$ is a graph which is obtained by deleting all non idempotent elements of $R$. Let $C$ be a subset of the vertex set in a graph $\Gamma$. Then $C$ is called a perfect code if for any $x, y \in C$ the union of the closed neighbourhoods of $x$ and $y$ gives the the vertex set and the intersection of the closed neighbourhoods of $x$ and $y$ gives the empty set. In this paper, the perfect codes in induced subgraphs of the unit graphs associated with the ring of integer modulo $n, \mathbb{Z}_{n}$ that has the vertex set as idempotent elements of $\mathbb{Z}_{n}$ are determined. The rings of integer modulo $n$ are classified according to their induced subgraphs of the unit graphs that accept a subset of a ring $\mathbb{Z}_{n}$ of different sizes as the perfect codes.
\end{abstract}

Key-Words: Ring of Integer Modulo $n$, Unit Graph, Induced Subgraph, Perfect Code

Received: March 29, 2021. Revised: August 12, 2021. Accepted: August 30, 2021. Published: September 5, 2021.

\section{Introduction}

Coding theory originated in the 1940 s, primarily with the work of Shannon [1]. He created the mathematical theory of information and proved that certain codes exist which can transmit information at near optimal rates with arbitrarily small chances of error. Later, in 1950, Hamming [2] introduced the theory of errorcorrecting codes. In the classical setting, every subset $C$ of $F_{q}^{n}$ is called a $q$-ary code, where $F_{q}^{n}$ is the set of all words of length $n$ over alphabet $F_{q}$ and every element of $C$ is called a code word. If a word $c$ is transmitted and $t$ errors occur, the received word $w$ will differ from $c$ in exactly $t$ bits. This is the distance between $c$ and $w$, and is called the Hamming distance. A $q$-ary code $C \subseteq F_{q}^{n}$ is called a perfect $t$ code if every word in $F_{q}^{n}$ is at distance not more than $t$ to exactly one code word of $C$ [3]. In view of the foregoing, mathematically a subset $C$ of a finite metric space can be thought as a perfect t-code [4] if the balls with radius $t$ centred in the $C$, form a partition of the metric space. Since a graph is a metric space under the usual graph distance; the distance of the vertex $r$ from the vertex $s$ in a graph $\Gamma$ is denoted by $d(r, s)$, and defined to be the shortest path between these two vertices of $\Gamma$ if a path exists between the vertices $r$ and $s$, while $d(r, s)$ is defined to be $\infty$ if no path exists between the vertices $r$ and $s$. Therefore, a graph $\Gamma$ with a distance function $d(r, s)$ satisfies all the conditions of a metric space. Based on the above view, the perfect $t$-codes can be defined in graphs; a subset $C$ of the vertex set, $V(\Gamma)$ is called a perfect $t$-code in $\Gamma$ if every vertex of $\Gamma$ is at distance not more than $t$ to exactly one vertex of $C$. Since 1973, perfect codes in graphs have been studied as a generalization of the perfect codes in the classical setting. Biggs [5] investigated the perfect codes in distance-transitive graph that admit non-trivial perfect codes, while Kratochvil [6] proved the non existence of perfect codes with size at least 2 (non-trivial perfect codes) in a complete bipartite graph. In the recent years, the study of perfect codes in graphs have been extended to the perfect codes in algebraic graphs. The problem when a subgroup of a group is a perfect code admitted by their associated graphs has gotten much attention. Huang et al. [7] established some sufficient and necessary conditions for the subgroup of a group to be perfect code admitted by the Cayley graphs, while Ma et al. [8] proved that in a Cayley graph all subgroups of a group are perfect codes if and only if the group does not contain elements of order four. In addition, it has been shown that for an abelian group $G$ its proper subgroup $K$ is considered as a perfect code of the group $G$ if and only if the $\mathrm{Syl}_{2} \mathrm{~K}$ is a perfect code of $\mathrm{Syl}_{2} \mathrm{G}$. In 2021, Zhang and Zhou [9] focused on some families of groups namely, 2-groups, metabelian groups, nilpotent groups and generalized dihedral groups of Cayley graphs and provided some conditions for their subgroups to be perfect codes. However, Chen et al. [10] determined the finite groups in which their associated Cayley graphs do not admit the non-trivial subgroup perfect codes. Therefore, the problem when a subset of a ring or a subring of a ring is a perfect code admitted by their associated graphs can attract notable attention which is an extension of the above stated problem.

Ashrafi et al. [11] constructed a unit graph by 
defining the elements of the ring $R$ as the vertex set and the vertex $r$ which is distinct from the vertex $s$ are adjacent if and only if $r+s$ is a unit of $R$. Some of the properties of this graph such as the girth [12], planarity [13], domination number [14], diameter [15] and Hamiltonian [16] have also been discovered.

The induced subgraph of a unit graph is denoted by $\Gamma^{\prime}(R)$, where the vertex set of $\Gamma^{\prime}(R)$ is the idempotent elements of $R=\mathbb{Z}_{n}, i d(R)$ and the vertex $r$ which is distinct from the vertex $s$ are adjacent if and only if $r+s$ is a unit of $R$. An element $r \in R$ is called an idempotent if $r^{2}=r$ [3].

In this paper, we determine the perfect codes in induced subgraphs of unit graphs by focusing on the theme: when a subset of the idempotent elements of a ring is a perfect code in induced subgraph of a unit graph. Starting in Section 2, we obtain results on the subset perfect codes of a ring $R=\mathbb{Z}_{n}$ over the induced subgraph of the unit graph. We show that the induced subgraph of a unit graph of a ring $R=\mathbb{Z}_{n}$ admit a perfect code of size 2 if $n$ is given as the product of two prime powers, where one of the prime is even (Theorem 2.1). In general, for the ring $R=\mathbb{Z}_{n}$ if $n$ is given as the product of $k$ factors of odd prime powers, we prove that the induced subgraphs of a unit graph admit the perfect code of size 1 (Theorem 2.2). We further prove a perfect code of size $2^{n-1}$ in induced subgraph of a unit graph when the ring $R$ is given as product of $n$ factors of $\mathbb{Z}_{p^{n}}$ (Theorem 2.3).

\section{Perfect codes in Induced Subgraph of Unit Graph}

In this section, some results are obtained on induced subgraph of a unit graph with vertex set as idempotent elements of $R=\mathbb{Z}_{n}$ which accept the subset $R=\mathbb{Z}_{n}$ as the perfect codes.

Theorem 2.1. Let $R=\mathbb{Z}_{n}$, where $n=$ $2^{m_{1}} p^{m_{2}}, m_{1}, m_{2} \geq 1$ and $p$ is an odd prime and $\Gamma^{\prime}(R)$ be the induced subgraph of $\Gamma(R)$. Then the following statements hold:

(i) $\Gamma^{\prime}(R)=K_{2,2} ; K_{2,2}$ is a bipartite graph with partite set of size 2 .

(ii) The subset $C$ of ring $R$ is the perfect code of size 2.

Proof. (i) Let $R=\mathbb{Z}_{n}$, where $n=$ $2^{m_{1}} p^{m_{2}}, m_{1}, m_{2} \geq 1$ and $p$ is an odd prime, then $R$ contains exactly four idempotent elements, that is $i d(R)=\left\{x_{i}: i=1,2,3,4\right\}$. Suppose that $x_{1}=0$ and $x_{2}=1$ are the trivial idempotent elements of $R$, then $x_{3}$ and $x_{4}$ are any two non-trivial idempotents of $R$. Since any non-trivial idempotent element is non-unit, therefore $x_{3}, x_{4} \notin U(R)$. According to the hypothesis of the theorem, $n=2^{m_{1}} p^{m_{2}}$, thus one of the non-trivial idempotent is a multiple of
2 and another one is a multiple of $p$. Suppose that $x_{3}$ is a multiples of 2 and $x_{4}$ is a multiple of $p$, then $x_{3}+x_{4}=n+1 \equiv 1 \bmod n$. This gives $x_{1}+x_{3} \notin U(R)$ and $x_{1}+x_{4} \notin U(R)$ which means that the vertex $x_{1}$ is not connected with the vertices $x_{3}$ and $x_{4}$. Since $x_{2} \in U(R)$, it yields that $x_{1}+x_{2} \in U(R)$ and $x_{2}+x_{3} \in U(R)$, that is, the vertex $x_{2}$ is connected with the vertices $x_{1}$ and $x_{3}$. Furthermore, since $x_{3}+x_{4}=n+1 \equiv 1 \bmod n$, thus $x_{3}+x_{4} \in U(R)$, meaning the vertex $x_{3}$ is connected with the vertex $x_{3}$. Hence, the vertex adjacency of $\Gamma^{\prime}(R)$ gives $\Gamma^{\prime}(R)$ to be a bipartite graph, i.e $\Gamma^{\prime}(R)=K_{2,2}$.

(ii) To prove the subset $C$ of ring $R$ is the perfect code of size 2, we obtain the closed neighbourhood of each vertex in $\Gamma^{\prime}(R)$ of radius 1 as in the following:

$$
\begin{aligned}
& S_{1}\left(x_{1}\right)=\left\{x_{1}, x_{2}\right\} \\
& S_{1}\left(x_{2}\right)=\left\{x_{1}, x_{2}, x_{3}\right\} \\
& S_{1}\left(x_{3}\right)=\left\{x_{2}, x_{3}, x_{4}\right\} \\
& S_{1}\left(x_{4}\right)=\left\{x_{3}, x_{4}\right\}
\end{aligned}
$$

From (1), (2), (3) and (4), it can be seen that (1) and (4) are the partitions of $V\left(\Gamma^{\prime}(R)\right)$, since $S_{1}\left(x_{1}\right) \cap$ $S_{1}\left(x_{4}\right)=\emptyset$ and $S_{1}\left(x_{1}\right) \cup S_{1}\left(x_{4}\right)=V\left(\Gamma^{\prime}(R)\right)$. Hence, $C=\left\{x_{1}, x_{4}\right\}$ is the perfect codes of size 2 in $\Gamma^{\prime}(R)$.

Example 2.1. Let $R=\mathbb{Z}_{100}$, where $100=2^{2} \cdot 5^{2}$.

Then $U(R)=\{1,3,7,9,11,13,17,19,21,23,27$, $29,31,33,37,39,41,43,47,49,51,53,57,59,61,63$, $67,69,71,73,77,79,81,83,87,89,91,93,97,99\}$ and $i d(R)=\{0,1,25,76\}$. By $\Gamma^{\prime}(R)$, $V\left(\Gamma^{\prime}(R)\right)=\{0,1,25,76\}$ and $E\left(\Gamma^{\prime}(R)\right)=$ $\{\{0,1\},\{1,76\},\{76,25\}\}$. Therefore, $\Gamma^{\prime}(R)$ is a bipartite graph with partite set of size 2 , given in Figure 2.1.

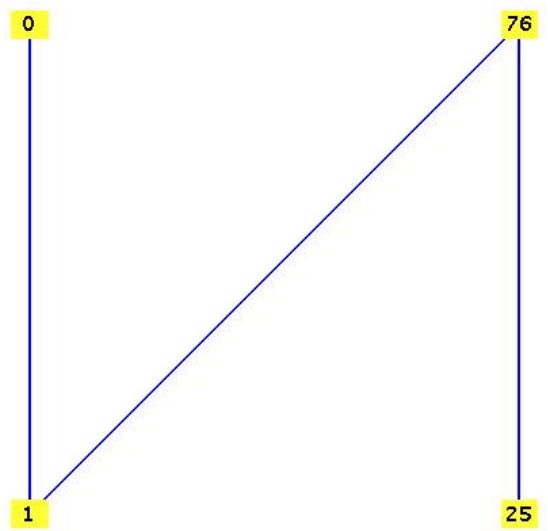

Figure 2.1: The induced subgraph of unit graph of $R=Z_{100}$. 
By Theorem 2.1, $C=\{0,25\}$ is the perfect code of size 2 in $\Gamma(R)$.

Theorem 2.2. Let $R=\mathbb{Z}_{n}$, where $n=$ $p_{1}^{m_{1}} p_{2}^{m_{2}}, \cdots p_{k}^{m_{k}}, m_{1}, m_{2}, \cdots, m_{k} \geq 1$ and $p_{i}$ are distinct odd primes and $\Gamma^{\prime}(R)$ be the induced subgraph of $\Gamma(R)$. Then the following statements hold:

(i) $\Gamma^{\prime}(R)$ contains finite number of triangles, $\left\{1, x_{i}, x_{j}\right\}$ as its subgraphs ;

(ii) The subset $C$ of a ring $R$ is the perfect code of size 1.

Proof. (i) Assume $R=\mathbb{Z}_{n}$, where $n=$ $p_{1}^{m_{1}} p_{2}^{m_{2}}, \cdots p_{k}^{m_{k}}, m_{1}, m_{2}, \cdots, m_{k} \geq 1$ and $p_{i}$ are distinct odd primes. Then $i d(R)=\left\{x_{i}: i=\right.$ $1,2,3, \cdots, k\}$. Since all non-trivial idempotent elements of $R$ are multiples of $p_{i}$, therefore the nontrivial idempotent elements are non-units. Let $x_{1}=0$ and $x_{2}=1$ are the trivial idempotents and $\left\{x_{j}\right.$ : $j=3,4, \cdots, k\}$ be the non-trivial idempotents. Then $x_{1}+x_{2} \in U(R), x_{1}+x_{j} \notin U(R)$ and $x_{2}+x_{j} \in$ $U(R)$. Similarly, $x_{i}+x_{k} \in U(R)$ for some nontrivial idempotents $x_{i}$ and $x_{k}$ if $x_{i}$ and $x_{k}$ are multiples of different primes. Therefore, $\Gamma^{\prime}(R)$ is a graph that contains finite number of triangles, $\left\{x_{2}, x_{i}, x_{j}\right\}$ as its subgraphs, where $x_{2}, x_{i}$ and $x_{j}$ are distinct vertices in $\Gamma^{\prime}(R)$.

(ii) Since all vertices in $\Gamma^{\prime}(R)$ are at distance not more than 1 to the vertex $x_{2}$, meaning that $S_{1}\left(x_{2}\right)=$ $V(\Gamma(R))$. Thus $C=\left\{x_{2}\right\}$ is the perfect code in $\Gamma^{\prime}(R)$.

Example 2.2. Let $R=\mathbb{Z}_{105}$, where $105=3 \cdot 5 \cdot 7$.

Then $U(R)=\{1,2,4,8,11,13,16,17,19,22,23,26$, $29,31,32,34,37,38,41,43,44,46,47,52,53,58,59$, $61,62,64,67,68,71,73,74,76,79,82,83,86,88,89$, $92,94,97,101,103,104\}$ and $i d(R)=\{0,1,15,21$,

$36,70,85,91\}$. By $\Gamma^{\prime}(R), V\left(\Gamma^{\prime}(R)\right)=\{0,1,15,21$, $36,70,85,91\}$ and $E\left(\Gamma^{\prime}(R)\right)=\{\{0,1\},\{1,15\}$, $\{1,21\},\{1,36\},\{1,70\},\{1,85\},\{1,91\},\{15,91\}$, $\{21,85\},\{36,70\},\{36,85\},\{85,91\}\}$. Therefore, $\Gamma^{\prime}(R)$ is a graph with 8 vertices and 12 edges, given in Figure 2.2.

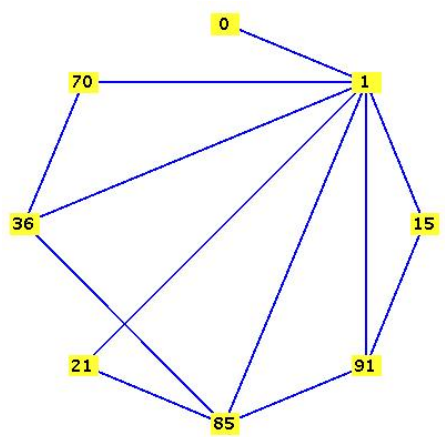

Figure 2.2: The induced subgraph of unit graph of $R=Z_{105}$.

By Theorem 2.2, $C=\{1\}$ is the perfect code of size 1 in $\Gamma(R)$.

In the next example, we show that if in the condition of Theorem 2.2, at least one of the $p_{i}$ be an even prime, then $\Gamma(R)$ does not accept the perfect codes.

Example 2.3. Let $R=\mathbb{Z}_{30}$, where $30=2 \cdot 3 \cdot 5$.

Then $U(R)=\{1,7,11,13,17,19,23,29\}$ and $i d(R)=\{0,1,6,10,15,16,21,25\}$. By $\Gamma^{\prime}(R)$,

$V\left(\Gamma^{\prime}(R)\right)=\{0,1,6,10,15,16,21,25\}$ and $E\left(\Gamma^{\prime}(R)\right)=\{\{0,1\},\{1,6\},\{1,10\},\{1,16\},\{6,25\}$ $\{10,21\},\{15,16\},\{16,21\},\{16,25\}\}$. Therefore, $\Gamma^{\prime}(R)$ is a bipartite graph with partite set of size 4, given in Figure 2.3.

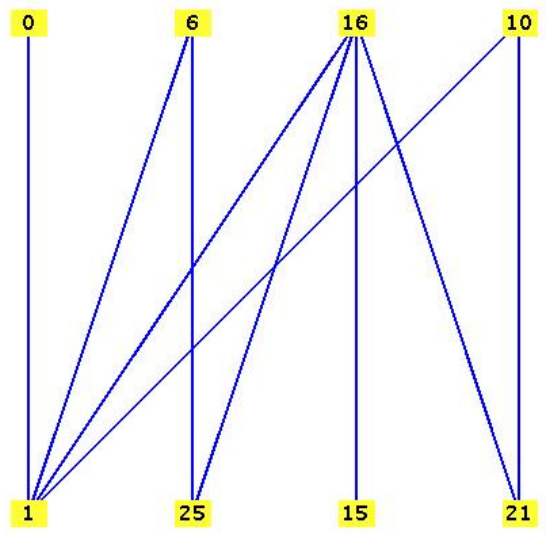

Figure 2.3: The induced subgraph of unit graph of $R=Z_{30}$.

To show no subset of the ring $R$ is a perfect code in $\Gamma^{\prime}(R)$, we obtain the closed neighbourhood of each vertex in $\Gamma^{\prime}(R)$ of radius 1 as in the following:

$$
\begin{aligned}
S_{1}(0) & =\{0,1\} \\
S_{1}(1) & =\{0,1,6,10,16\} \\
S_{1}(6) & =\{1,6,25\} \\
S_{1}(10) & =\{1,10,21\} \\
S_{1}(15) & =\{15,16\} \\
S_{1}(16) & =\{1,15,16,21,25\} \\
S_{1}(21) & =\{10,16,21\} \\
S_{1}(25) & =\{6,16,25\}
\end{aligned}
$$

The closed neighbourhoods of the vertices $0,1,6,10,15,16,21$ and 25 show that the union of no distinct neighbourhoods gives the whole vertex set. Consequently, no subset of the ring $R$ is a perfect code in $\Gamma^{\prime}(R)$. 
Theorem 2.3. Let $R=\mathbb{Z}_{p^{m}} \times \mathbb{Z}_{p^{m}} \times \mathbb{Z}_{p^{m}} \times \cdots \times \mathbb{Z}_{p^{m}}$ with $n$ factors and $\Gamma^{\prime}(R)$ be the induced subgraph of $\Gamma(R)$. Then the following statements hold:

(i) $\Gamma^{\prime}(R)=\bigcup_{k=1}^{2^{n-1}} K_{2}$.

(ii) The subset $C$ of ring $R$ is a perfect code of size $2^{n-1}$

Proof. Assume $R=\mathbb{Z}_{p^{m}} \times \mathbb{Z}_{p^{m}} \times \mathbb{Z}_{p^{m}} \times \cdots \times \mathbb{Z}_{p^{m}}$ with $n$ factors. Then $i d(R)=\left\{x_{i}: i=\right.$ $\left.1,2,3, \cdots, 2^{n}\right\}$. Let $x_{1}=(0,0,0, \cdots, 0)$, $x_{2}=(0,0,0, \cdots, 1), \cdots, x_{2^{n}}=(1,1,1, \cdots, 1)$. By $\Gamma^{\prime}(R), V\left(\Gamma^{\prime}(R)\right)=i d(R)$ and two distinct vertices $x_{i}$ and $x_{j}$ are connected if and only if $x_{i}+x_{j} \in U(R)$. This gives that $E\left(\Gamma^{\prime}(R)\right)=$ $\left\{\left\{x_{1}, x_{2^{n}}\right\},\left\{x_{2}, x_{2^{n}-1}\right\}, \cdots,\left\{x_{2^{n-1}}, x_{2^{n-1}+1}\right\}\right\}$.

Hence, $\Gamma^{\prime}(R)=\bigcup_{k=1}^{2 n-1} K_{2}$.

To prove the subset $C$ of ring $R$ is a perfect code of size $2^{n-1}$, we determine the closed neighbourhood of all vertices with radius 1 in $\Gamma^{\prime}(R)$ as in the following:

$$
\begin{aligned}
S_{1}\left(x_{1}\right) & =\left\{x_{1}, x_{2^{n}}\right\}=S_{1}\left(x_{2^{n}}\right) \\
S_{1}\left(x_{2}\right) & =\left\{x_{2}, x_{2^{n}-1}\right\}=S_{1}\left(x_{2^{n}-1}\right) \\
S_{1}\left(x_{3}\right) & =\left\{x_{3}, x_{2^{n}-2}\right\}=S_{1}\left(x_{2^{n}-2}\right) \\
\vdots & \\
S_{1}\left(x_{2^{n-1}}\right) & =\left\{x_{2^{n-1}}, x_{2^{n-1}}+1\right\}=S_{1}\left(x_{2^{n-1}+1}\right)
\end{aligned}
$$

The above results show that $S_{1}\left(x_{1}\right), S_{1}\left(x_{2}\right), S_{1}\left(x_{3}\right), \cdots, S_{1}\left(x_{2^{n-1}}\right) \quad$ partition the vertex set, $V\left(\Gamma^{\prime}(R)\right)$. Hence, the subset $C=\left\{x_{1}, x_{2}, x_{3}, \cdots, x_{2^{n-1}}\right\}$ of ring $R$ is a perfect code of size $2^{n-1}$.

\section{Conclusion}

In this paper, by the idea of a unit graph, its induced subgraphs are constructed according to the unit and idempotent elements of the ring of integer modulo $n$. The results that have been obtained represent the types of the induced subgraphs of the unit graphs as well as the perfect codes over these graphs. The results show that if $R=\mathbb{Z}_{n}$, where $n=2^{m_{1}} p^{m_{2}}, m_{1}, m_{2} \geq 1$ and $p$ is an odd prime, then the perfect code accepted by $\Gamma^{\prime}(R)$ is of size 2 , while if $R=\mathbb{Z}_{n}$, where $n=$ $p_{1}^{m_{1}} p_{2}^{m_{2}}, \cdots p_{k}^{m_{k}}, m_{1}, m_{2}, \cdots, m_{k} \geq 1$ and $p_{i}$ are distinct odd primes, then the perfect code accepted by $\Gamma^{\prime}(R)$ is of size 1 . However, if $R=\mathbb{Z}_{p^{m}} \times \mathbb{Z}_{p^{m}} \times$ $\mathbb{Z}_{p^{m}} \times \cdots \times \mathbb{Z}_{p^{m}}$, then the perfect code accepted by $\Gamma^{\prime}(R)$ is of size $2^{n-1}$. According to the existing literatures on perfect codes in graphs of groups, this research which is an extension of the previous studies present the subset perfect codes of the ring $R=\mathbb{Z}_{n}$. Therefore, this research open up many research areas to determine the subset or subring perfect codes of rings over the existence graphs associated with rings. Referring to the theory of error correcting codes in coding theory, a subset of the vertices of a graph is called t-error correcting code if the minimum distance between the two elements in the subset is at least $2 t+1$. Thus, as an application, the findings of this research might be helpful on single-error correcting codes since the minimum distance between the two elements of the perfect code $C$ is at least 3 .

\section{References:}

[1] C. E. Shannon, A mathematical theory of communication, The Bell system technical journal, Vol. 27, No. 3, 1948, pp. 379-423.

[2] R. W. Hamming, Error detecting and error correcting codes, The Bell system technical journal, Vol. 29, No. 2, 1950, pp. 147-160.

[3] W. K. Nicholson, Introduction to abstract algebra, John Wiley Sons, 2012.

[4] J. H. Van Lint, A survey of perfect codes, The Rocky Mountain Journal of Mathematics, Vol. 5, No. 2, 1975, pp. 199-224.

[5] N. Biggs, Perfect codes in graphs, Journal of Combinatorial Theory, Series B, Vol. 15, No. 3, 1973, pp. 289-296.

[6] J. Kratochvil, Perfect codes over graphs, Journal of Combinatorial Theory, Series B, Vol. 40, No. 2, 1986, pp. 224-228.

[7] H. Huang, B. Xia, and S. Zhou, Perfect codes in Cayley graphs, SIAM Journal on Discrete Mathematics, Vol. 32, No. 1, 2018, pp. 548-559.

[8] X. Ma, G. L. Walls, K. Wang, and S. Zhou, Subgroup perfect codes in Cayley graphs, SIAM Journal on Discrete Mathematics, Vol. 34, No. 3, 2020, pp. 1909-1921.

[9] J. Zhang and S. Zhou, On subgroup perfect codes in Cayley graphs, European Journal of Combinatorics, Vol. 91, 2021, p. 103228.

[10] J. Chen, Y. Wang, and B. Xia, Characterization of subgroup perfect codes in Cayley graphs, Discrete Mathematics, Vol. 343, No. 5, 2021, p. 111813.

[11] N. Ashrafi, H. Maimani, M. Pournaki, and S. Yassemi, Unit graphs associated with rings, Communications in Algebra, Vol. 38, No. 8, 2010, pp. 2851-2871.

[12] H. Su and Y. Zhou, On the girth of the unit graph of a ring, Journal of Algebra and Its Applications, Vol. 13, No. 2, 2014, pp. 1-12. 
[13] H. Su, G. Tang, and Y. Zhou, Rings whose unit graphs are planar, Publ. Math. Debrecen, Vol. 86, No. 3-4, 2015, pp. 363-376.

[14] S. Kiani, H. Maimani, M. Pournaki, and S. Yassemi, Classification of rings with unit graphs having domination number less than four, Rend. Sem. Mat. Univ. Padova, Vol. 133, 2015, pp. 173-195.
[15] H. Su and Y. Wei, The diameter of unit graphs of rings, Taiwanese Journal of Mathematics, Vol. 23, No. 1, 2019, pp. 1-10.

[16] H. R. Maimani, M. Pournaki, and S. Yassemi, Necessary and sufficient conditions for unit graphs to be Hamiltonian, Pacific journal of mathematics, Vol. 249, No. 2, 2011, pp. 419429.

\section{Creative Commons Attribution License 4.0 (Attribution 4.0 International, CC BY 4.0)}

This article is published under the terms of the Creative Commons Attribution License 4.0

https://creativecommons.org/licenses/by/4.0/deed.en_US 